\section{Distribuição espacial e temporal da tuberculose em indígenas e não indígenas de Rondônia, Amazônia Ocidental, Brasil}

\author{
Spatial and temporal distribution of tuberculosis in \\ indigenous and non-indigenous of Rondônia State, \\ Western Amazon, Brazil
}

Programa Nacional de
Controle da Tuberculose,
Ministério da Saúde, Brasília,
Brasil.
2 Escola Nacional de Saúde
Pública Sergio Arouca,
Fundação Oswaldo Cruz,
Rio de Janeiro, Brasil.
Correspondência
P. C. Basta
Departamento de Endemias
Samuel Pessoa, Escola
Nacional de Saúde Pública
Sergio Arouca, Fundação
Oswaldo Cruz.
Rua Leopoldo Bulhões 1480,
Rio de Janeiro, RJ 21041-210,
Brasil.
paulobasta@gmail.com

Abstract

This study analyzed the spatial and temporal distribution of crude and adjusted rates of incidence of tuberculosis (TB) between 1997 and 2006, identifying areas of greatest risk to the indigenous and non-indigenous population of Rondônia State, Brazil. An ecological study was conducted analyzing municipalities and Indian reserves, using the local empirical Bayesian method. The crude average rate of incidence of $T B$ among the non-indigenous population was 35.6/100,000 inhabitants, while for the indigenous population it was 415.0/100,000. Rates greater than 600/100,000 were reported in the Karipuna, Sete de Setembro, Igarapé, Ribeirão and Karitiana reserves. We observed a greater number of cases in under 15 year-olds with little schooling in contrast to the situation in the nonindigenous population. After making adjustments, the rates in some Indian reserves exceeded 240/100,000 inhabitants, while in coinciding municipalities incidence was between the range of 61-120/100,000. The Bayesian method led to decreased overall heterogeneity in rates. Evidence suggests that the indigenous population is more vulnerable to contracting $T B$ and highlighted areas that require further attention to ensure the adequate control of TB in Rondônia.

Tuberculosis; Spacial Analysis; Population Surveillance; South American Indians
Tatiana Eustáquia Magalhães de Pinho Melo 1 Ana Paula da Costa Resendes 2 Reinaldo Souza-Santos 2 Paulo Cesar Basta 2

\section{Introdução}

Com exceção dos estados do Rio Grande do Norte e Piauí, os povos indígenas estão presentes em praticamente todo território brasileiro ${ }^{1}$. De acordo com dados do Sistema de Informação da Atenção à Saúde Indígena (SIASI) da Fundação Nacional de Saúde (FUNASA), existem no Brasil 538.154 indígenas, representantes de 210 povos, falantes de mais de 170 línguas, residentes em 4.413 aldeias, distribuídas em 615 Terras Indígenas (TI), localizadas em 432 municípios 2 . Aproximadamente 120 mil indígenas vivem ainda em áreas urbanas e nas capitais dos estados 1,2,3.

As TI pertencentes à Amazônia Legal representam 98,63\% da extensão total das TI no país, enquanto $1,37 \%$ restante espalha-se ao longo das regiões Sudeste, Sul, Nordeste e Centro-oeste. Vale lembrar que em relação à demarcação e homologação, as TI no Brasil encontram-se em diferentes estágios de regularização fundiária 1.

No que diz respeito ao cenário de adoecimento e morte, o perfil epidemiológico dos povos indígenas é marcado por elevadas taxas de incidência e de mortalidade por doenças infecciosas e parasitárias, com destaque para infecções respiratórias, diarreia, malária e tuberculose (TB) 4,5 .

Nas últimas décadas, houve um importante incremento no número de instituições e pesquisadores interessados no tema da TB entre os povos indígenas no Brasil. A maioria dos estu- 
dos recentes concentra-se na região Amazônica $6,7,8,9,10,11,12,13,14$ e revela que, apesar das altas coberturas vacinais pela BCG, a incidência da doença é muito elevada (não raro atingindo cifras 40 vezes superiores às médias nacionais) $\mathrm{e}$ a prevalência de infecção é bastante expressiva (com cerca de 1/3 dos grupos investigados apresentando fortes reações à Prova Tuberculínica). Esses dados em conjunto levaram o Programa Nacional de Controle da Tuberculose (PNCT) a considerar os indígenas como populações especiais 15 .

Para o Estado de Rondônia em particular, nos dias atuais, já se dispõe de conhecimento sobre a epidemiologia da TB entre os indígenas. Por meio da análise dos casos notificados no SINAN estadual, Escobar et al. 7 asseguravam que na década de 1990 os indígenas apresentavam dez vezes mais chance de adoecer e morrer de TB que outros segmentos da população. Aprofundando o debate, Basta et al. ${ }^{9}$ constataram incidência média de TB, no período 1991-2002, acima de 2.500/100.000 entre os indígenas da etnia Suruí. Outro estudo baseado na busca ativa de sintomáticos respiratórios revelou a presença de uma cepa de Mycobacterium tuberculosis resistente à rifampicina e à isoniazida, demonstrou o envolvimento de quatro diferentes tipos de micobactérias na transmissão da doença e detectou ainda a presença de micobactérias ambientais em $12,8 \%$ das amostras de escarro examinadas nas aldeias analisadas 16,17 .

Apesar do crescente uso do geoprocessamento para análise da distribuição espacial da TB no Brasil 18,19,20,21,22, apenas um único trabalho foi localizado nas bases indexadas com dados referentes às notificações de tuberculose entre os indígenas no país 23 . Logo, o objetivo deste estudo foi analisar a distribuição espacial e temporal das taxas brutas e ajustadas de incidência de TB, no período compreendido entre 1997 a 2006, identificando áreas de maior risco nas populações indígena e não indígena do Estado de Rondônia, segundo TI e municípios.

\section{Material e métodos}

Trata-se de um estudo ecológico de dados agregados por áreas, tendo como unidades de análise os municípios e as TI do Estado de Rondônia.

O Estado de Rondônia, cuja capital é a cidade de Porto Velho, encontra-se localizado na Região Norte do país, tendo como fronteira: Bolívia (S e O), os estados do Acre (O), Amazonas (N) e Mato Grosso (L). Possui área equivalente a 237.590,864km², 52 municípios, população estimada para 2010 de 1.560.501 (http://www.ibge. gov.br/estadosat/perfil.php?sigla=ro, acessado em 10/Jan/2011). Possui 20 TI homologadas e uma em identificação, distribuídas entre 28 etnias 1 (http://www.funai.gov.br/mapas/fr_ma pa_endereco.htm, acessado em 01/Out/2008) (Figura 1). Comporta dois Distritos Sanitários Especiais Indígenas (DSEI), Porto Velho e Vilhena, que abrangem em sua extensão territorial parte dos estados de Mato Grosso e Amazonas (http:// sis.funasa.gov.br/portal/mapa_dsei.asp, acessado em 18/Out/2008).

Os dados referentes aos casos de TB, notificados no período de 1997 a 2006, foram obtidos junto ao Sistema de Informação de Agravos de Notificação (SINAN) gerenciado pela Coordenação Estadual do Controle de Hanseníase e Tuberculose da Secretaria Estadual de Saúde do Estado de Rondônia.

Para o cálculo das taxas de incidência, foi considerada como caso novo de TB toda notificação em que a variável "tipo de entrada" estivesse preenchida com as categorias "caso novo" ou "não sabe" 24.

Foram incluídos todos os casos novos de TB, em residentes nos municípios do Estado de Rondônia, disponíveis na base do SINAN, no período de $1^{\circ}$ de janeiro de 1997 a 31 de dezembro de 2006. Foram excluídos os registros duplicados e aqueles em que a situação de encerramento foi "mudança de diagnóstico".

Os casos novos foram analisados de forma agrupada quanto à classificação: indígena e não indígena. No grupo de não indígenas, foram incluídas as demais categorias de raça/cor, a saber: branco, preto, amarelo, pardo e ignorado.

Uma particularidade observada entre os indígenas em Rondônia foi que habitualmente o sobrenome se sobrepõe à etnia (p. ex.: José Suruî). Em decorrência disso, e devido ao preenchimento insatisfatório do campo raça/cor, o procedimento de classificação étnica dos doentes se deu com base na consulta ao campo de identificação do nome-sobrenome, e nos campos referentes ao endereço de residência e da unidade notificadora, disponíveis no SINAN. Tal método foi aplicado em outros contextos e é utilizado em pesquisas epidemiológicas de recorte étnico/racial 25,26.

A classificação aqui empregada foi realizada de forma independente por um dos autores e um colaborador que têm experiência prévia com trabalhos dessa natureza. Para avaliação da concordância, utilizou-se a estatística kappa. A concordância foi considerada quase perfeita $(0,97)$, segundo a classificação proposta por Landis \& Koch 27. Nos casos em que houve discordância, elas foram resolvidas por consenso após consulta complementar realizada aos profissionais dos DSEI Porto Velho e Vilhena. 

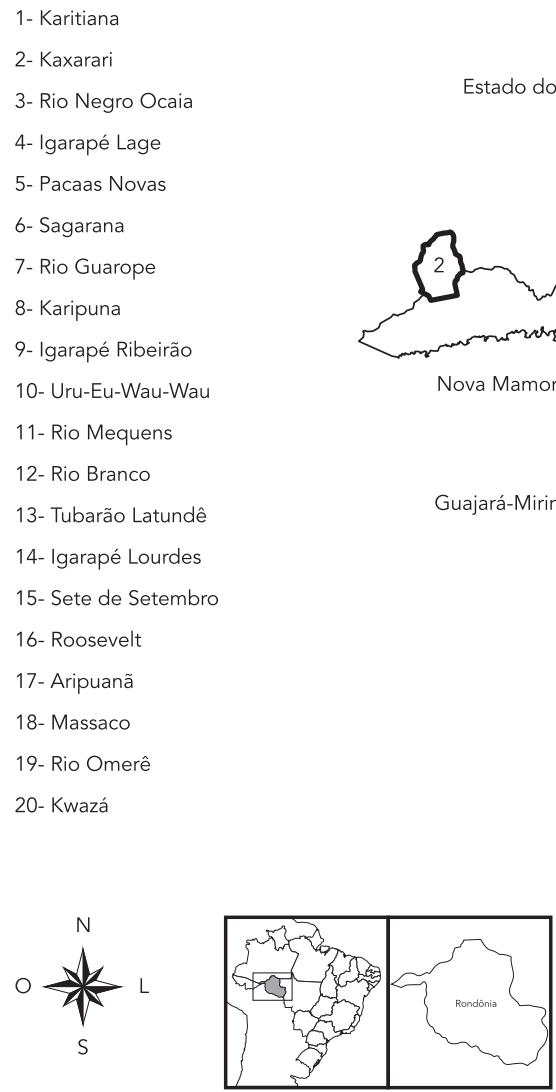

Os dados populacionais das TI referentes ao ano 2000 se originam no documento Povos Indígenas no Brasil 2001/2005, produzido pelo Instituto Socioambiental 1. Para os demais anos, realizaram-se estimativas populacionais mediante cálculos de progressão geométrica com taxa média anual de crescimento de $4,5 \%$ ao ano, conforme assinala estudo realizado pelo Instituto Brasileiro de Geografia e Estatística (IBGE) 28. Os dados populacionais referentes aos municípios foram estimados segundo o Censo Demográfico 2000.

As malhas digitais cartográficas dos municípios e TI do Estado de Rondônia foram adquiridas junto ao IBGE (http://www.ibge.gov.br/ mapas_ibge/bases_malhas.php, acessado em 18/Out/2008) e ao Departamento de Assuntos
Fundiários (DAF) da Fundação Nacional do Índio (FUNAI; http://www.funai.gov.br, acessado em 04/Jan/2009), respectivamente.

A localização dos casos de TB, de acordo com a TI de origem, foi feita com consulta aos campos "nome" e "endereço" no módulo demográfico do SIASI, disponível na Coordenação Regional da Fundação Nacional de Saúde em Rondônia. Por essa consulta foi possível identificar as aldeias e as TI, sem discriminar o município de residência, pois diversas TI abrangem mais de um município em sua extensão territorial. Já para localização dos casos de TB na população não indígena foi consultado o campo município de residência no SINAN.

Foi realizada uma análise descritiva dos casos de TB segundo sexo, faixa etária, escolaridade 
e raça/cor, e construída uma série histórica das taxas de incidência bruta de TB na população indígena e não indígena para o período de 1997 a 2006.

Para o ajuste das taxas, as malhas digitais cartográficas das TI $(\mathrm{n}=20)$ e dos municípios ( $n=52$ ) foram agregadas, perfazendo 72 unidades territoriais. Após a agregação, foram calculadas taxas ajustadas para quatro períodos (1997-1999, 2000-2002, 2003-2006 e 1997-2006), utilizando-se o método bayesiano empírico local. A ideia central desse método é promover uma suavização nas medidas de ocorrência de um evento/agravo em áreas com pequenas populações. Nessas situações pode haver flutuação aleatória nas medidas, fato que pode enviesar as estimativas. $\mathrm{O}$ método parte do pressuposto de que um evento ocorrido numa área pode influenciar e pode ser influenciado pelo que ocorre em sua vizinhança 29,30.

Pelo fato de a TB ser uma doença de transmissão interpessoal, e admitindo que ocorre/ ocorreu contato entre as populações indígena e não indígena, circunvizinhas, utilizou-se para o ajuste das taxas de incidência uma matriz de vizinhança por distância. Tendo em conta a distância média existente entre as TI e os centros urbanos de referência para diagnóstico e tratamento da TB, usou-se para construção da matriz de vizinhança um raio de $100 \mathrm{~km}$.

Posteriormente, foram construídos mapas temáticos das taxas brutas, segundo municípios e TI isolados, e das taxas ajustadas, segundo municípios e TI agregados, para os quatro períodos supracitados.

Foi considerada neste artigo a expressão "municípios de referência” para os municípios coincidentes (que contêm ou estão contidos nas TI).

Os dados foram estruturados em planilhas eletrônicas (Excel, Microsoft Corp., Estados Unidos) e analisados nos programas SPSS, versão 9.0 (SPSS Inc., Chicago, Estados Unidos), e TerraView versão 3.6.0 (Instituto Nacional de Pesquisas Espaciais, São José dos Campos, Brasil).

Este estudo é parte de uma investigação mais ampla denominada Tuberculose entre os Povos Indígenas de Rondônia, que foi aprovada pelo Comitê de Ética em Pesquisa da Escola Nacional de Saúde Pública Sergio Arouca (CEP/ENSP; parecer $n^{\circ}$. 14/07).

\section{Resultados}

No período de 1997-2006, foram notificados 5.264 casos novos de TB entre a população residente (indígenas e não indígenas) no Estado de Rondô- nia, com média anual de 526 notificações. Entre a população não indígena foram contabilizados 95\% de todos os casos $(\mathrm{n}=4.986)$, com média anual de 499 notificações. Na população indígena foram registrados 278 casos novos no período ( $5 \%$ do total), com média anual de 28 casos.

A taxa média bruta de incidência de TB para os não indígenas foi 35,57/100.000, enquanto para os indígenas foi 415,03/100.000 habitantes. Na Tabela 1 é possível verificar a ocorrência de casos assim como as taxas médias brutas de incidência de TB, agrupadas em quatro períodos (1997-1999, 2000-2002, 2003-2006 e 1997-2006), de acordo com as TI em Rondônia. Destaca-se que as TI Karipuna, Sete de Setembro, Igarapé Ribeirão e Karitiana apresentaram incidências acima de 600/100.000.

A variação nas taxas brutas de incidência foi pequena para a população não indígena. Entretanto, entre os indígenas, encontrou-se importante flutuação que pode ser em razão das pequenas populações residentes em algumas TI (Tabela 1, Figura 2).

Ao analisar a distribuição dos casos de TB segundo sexo, foi constatado predomínio em homens, tanto para população indígena (56,5\%; $\chi^{2}=5,12 ; 1$ gl; $\mathrm{p}=0.02$ ) quanto para população não indígena $\left(62,8 \% ; \chi^{2}=327,31 ; 1\right.$ gl; $\left.\mathrm{p}<0,001\right)$ (Tabela 2).

Em relação à variável faixa etária, 35,6\% e $24,5 \%$ dos casos de TB na população indígena foram registrados em menores de 15 anos e em adultos jovens de 15 a 24 anos, respectivamente. Para a população não indígena, as notificações em menores de 15 anos corresponderam a apenas $4,5 \%$, e os maiores percentuais foram encontrados nas faixas etárias de 15 a 24, 25 a 34 e 35 a 44 anos, com 19,6\%, 22,3\% e 19,4\%, respectivamente (Tabela 2).

A variável raça/cor foi preenchida somente em $51,1 \%$ dos casos de indígenas e em apenas $46,2 \%$ das notificações de não indígenas (Tabela 2).

No que diz respeito à escolaridade, chama a atenção que em $34,2 \%$ dos casos notificados em indígenas a variável foi preenchida com a categoria nenhuma escolaridade. Além disso, em $27,7 \%$ dos casos indígenas, tal variável foi preenchida com a categoria "não se aplica”, totalizando $61,9 \%$ dos casos de TB em indígenas com baixo ou nenhum nível de instrução formal. Em contrapartida, na população não indígena foi possível verificar que $43,3 \%$ dos casos apresentavam de 4 a 7 anos de estudo (Tabela 2). 
Número de casos, população e taxa de incidência bruta de tuberculose (TB), segundo Terras Indígenas, Estado de Rondônia, Brasil, $1997-2006$.

\begin{tabular}{|c|c|c|c|c|c|c|c|c|c|c|c|c|}
\hline \multirow[t]{2}{*}{ Terra Indígena } & \multicolumn{4}{|c|}{ Casos de TB } & \multicolumn{4}{|c|}{ População } & \multicolumn{4}{|c|}{ Taxa de incidência bruta } \\
\hline & $\begin{array}{l}1997- \\
1999\end{array}$ & $\begin{array}{l}2000- \\
2002\end{array}$ & $\begin{array}{l}2003- \\
2006\end{array}$ & Total & $\begin{array}{l}1997- \\
1999\end{array}$ & $\begin{array}{l}2000- \\
2002\end{array}$ & $\begin{array}{l}2003- \\
2006\end{array}$ & Total & $\begin{array}{l}1997- \\
1999\end{array}$ & $\begin{array}{c}2000- \\
2002\end{array}$ & $\begin{array}{l}2003- \\
2006\end{array}$ & Média \\
\hline Igarapé Lage & 9 & 6 & 8 & 23 & 1.193 & 1.362 & 2.119 & 4.675 & 754,1 & 440,6 & 377,5 & 492,0 \\
\hline Igarapé Lourdes & 3 & 9 & 5 & 17 & 1.388 & 1.583 & 2.464 & 5.435 & 216,2 & 568,4 & 202,9 & 312,8 \\
\hline Igarapé Ribeirão & 8 & 4 & 6 & 18 & 536 & 612 & 952 & 2.100 & 1492,4 & 653,9 & 630,3 & 857,3 \\
\hline Karipuna & 0 & 0 & 2 & 2 & 13 & 15 & 24 & 52 & 0,0 & 0,0 & 8508,5 & 3857,6 \\
\hline Karitiana & 6 & 6 & 7 & 19 & 706 & 764 & 1.254 & 2.724 & 850,0 & 785,3 & 558,4 & 697,6 \\
\hline Kaxarari & 0 & 0 & 0 & 0 & 682 & 778 & 1.211 & 2.671 & 0,0 & 0,0 & 0,0 & 0,0 \\
\hline Kwazá do Rio São & 0 & 0 & 0 & 0 & 66 & 75 & 117 & 258 & 0,0 & 0,0 & 0,0 & 0,0 \\
\hline \multicolumn{13}{|l|}{ Pedro } \\
\hline Massaco & 0 & 0 & 0 & 0 & 406 & 459 & 706 & 1.571 & 0,0 & 0,0 & 0,0 & 0,0 \\
\hline Pacaas Novas & 21 & 6 & 9 & 36 & 2698 & 3.079 & 4.791 & 10.568 & 778,4 & 194,9 & 187,8 & 340,7 \\
\hline Parque do Aripuanã & 0 & 0 & 0 & 0 & 938 & 1.070 & 1.665 & 3.672 & 0,0 & 0,0 & 0,0 & 0,0 \\
\hline Rio Branco & 2 & 7 & 0 & 9 & 1.145 & 1.306 & 2.033 & 4.485 & 174,7 & 535,8 & 0,0 & 200,7 \\
\hline Rio Guaporé & 1 & 1 & 4 & 6 & 1.299 & 1.483 & 2.308 & 5.089 & 77,0 & 67,4 & 173,3 & 117,9 \\
\hline Rio Mequéns & 0 & 0 & 0 & 0 & 234 & 267 & 416 & 917 & 0,0 & 0,0 & 0,0 & 0,0 \\
\hline Rio Negro/Ocaia & 4 & 4 & 1 & 9 & 593 & 677 & 1.054 & 2.324 & 674,1 & 590,7 & 94,9 & 387,2 \\
\hline Rio Omerê & 0 & 0 & 0 & 0 & 24 & 28 & 43 & 95 & 0,0 & 0,0 & 0,0 & 0,0 \\
\hline Roosevelt & 2 & 2 & 2 & 6 & 1.321 & 1.507 & 2.345 & 5.173 & 151,5 & 132,7 & 85,3 & 116,0 \\
\hline Sagarana & 1 & 0 & 3 & 4 & 195 & 223 & 347 & 764 & 512,5 & 0,0 & 865,7 & 523,3 \\
\hline Sete de Setembro & 18 & 45 & 52 & 115 & 2.316 & 2.643 & 4.113 & 9072 & 777,2 & 1702,7 & 1264,3 & 1267,7 \\
\hline Tubarão/Latundê & 0 & 1 & 0 & 1 & 397 & 453 & 705 & 1.555 & 0,0 & 220,7 & 0,0 & 64,3 \\
\hline Uru-Eu-Wau-Wau & 1 & 1 & 2 & 4 & 413 & 471 & 733 & 1.616 & 242,4 & 212,4 & 273,0 & 247,5 \\
\hline Total & 76 & 92 & 101 & 269 & 16.562 & 18.854 & 29.399 & 64.815 & 458,9 & 488,0 & 343,6 & 415,0 \\
\hline Desaldeados & 1 & 8 & 0 & 9 & & & & & & & & \\
\hline Estado Rondônia & 1.608 & 1.483 & 1.895 & 4.986 & 3.992 .255 & 4.171 .806 & 5.855 .295 & 14.019 .356 & 40,3 & 35,6 & 32,4 & 35,6 \\
\hline
\end{tabular}

Incidência bruta de tuberculose na população não indígena de Rondônia, segundo município de residência

A análise espacial das taxas brutas, segundo município de residência, demonstrou que, no triênio 1997-1999, Porto Velho, Guajará-Mirim e Pimenteiras do Oeste destacaram-se dentre os demais por apresentarem incidência na faixa de 61-120 casos novos por 100.000 habitantes (Figura 3a).

Em relação ao triênio 2000-2002, observou-se redução na incidência para faixa de 0-30/100.000 habitantes em Pimenteiras do Oeste. No entanto, Porto Velho e Guajará-Mirim permaneceram com a incidência estabilizada na faixa de 61120/100.000 habitantes (Figura 3b).

No quadriênio correspondente ao período 2003-2006, verificou-se redução em Porto Velho e aumento das taxas nos municípios de Candeias do Jamari e Vale do Anari. O Município de Guajará-Mirim continuou com a taxa estabilizada em altos patamares na faixa de 61-120/100.000 habitantes (Figura 3c).

A despeito das flutuações observadas nos três períodos estudados, a análise do decênio 19972006 indicou que Guajará-Mirim e Porto Velho foram os municípios nos quais se pode constatar as maiores incidências brutas de TB (61-120 casos novos por 100.000 habitantes) em Rondônia (Figura 3d).

\section{Incidência bruta de tuberculose na população indígena de Rondônia, segundo Terras Indígenas}

A análise da distribuição espacial dos casos de TB segundo as TI de Rondônia revelou que no triênio 1997-1999 as TI Karitiana, Uru-Eu-Wau-Wau, Igarapé Ribeirão, Igarapé Lage, Rio Negro Ocaia, Pacaas Novas, Sagarana e Sete de Setembro apresentaram taxas brutas superiores a 241/100.000 habitantes. As TI Rio Branco, Igarapé Lourdes e 
Taxas de incidência bruta da tuberculose por 100.000 habitantes segundo população indígena e população não indígena do Estado de Rondônia, Brasil, 1997 a 2006.

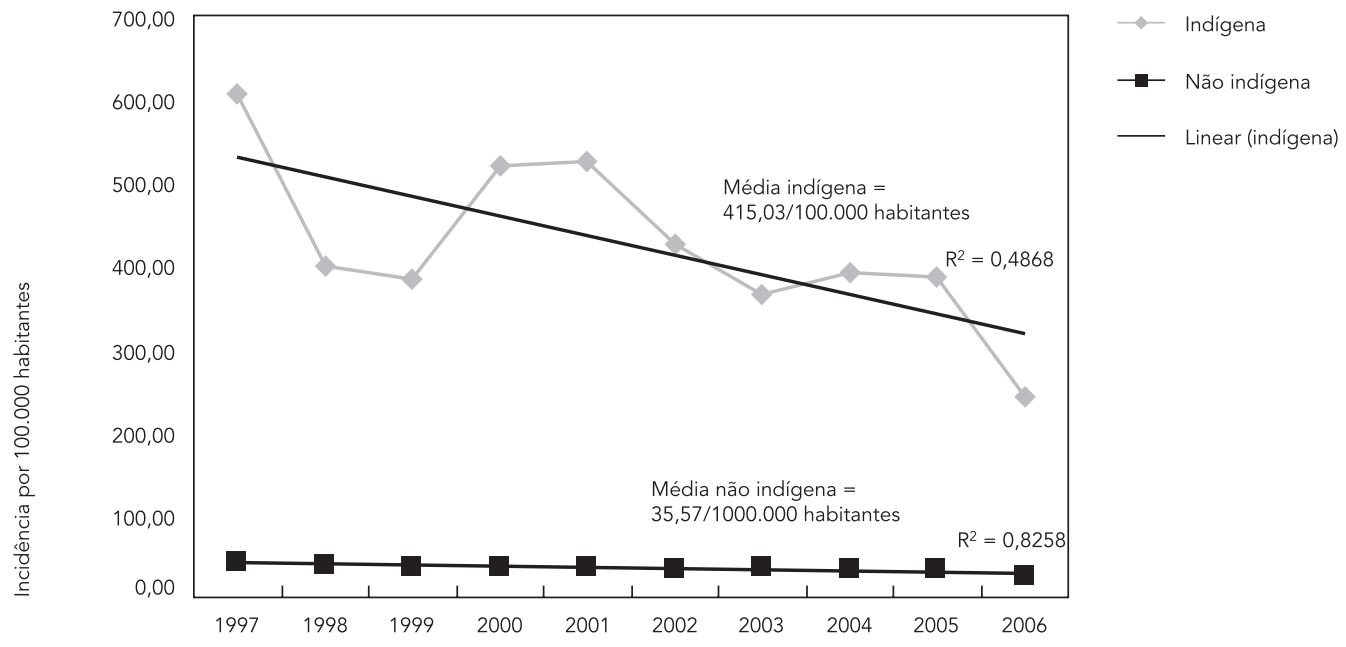

Roosevelt apresentaram incidências na faixa de 121-240/100.000. Já a TI Rio Guaporé apresentou incidência oscilando entre 61-120/100.000, enquanto as demais TI apresentaram taxas brutas inferiores a 31/100.000 habitantes (Figura 4a).

No triênio 2000-2002, as TI Uru-Eu-WauWau, Pacaas Novas e Sagarana apresentaram uma situação menos grave, com ligeira redução nas taxas, à medida que a incidência na TI Roosevelt permaneceu estável. Contudo, houve aumento na incidência na TI Tubarão Latundê (121-240/100.000) e nas TI Rio Branco e Igarapé Lourdes (> 241/100.000) (Figura 4b).

A análise do quadriênio correspondente ao período 2003-2006 mostrou que as TI Karitiana, Igarapé Ribeirão, Igarapé Lage e Sete de Setembro permaneceram com incidências superiores a 241/100.000 habitantes. A TI Pacaas Novas permaneceu com taxa na faixa de 121-240/100.000 habitantes. Nas TI Uru-Eu-Wau-Wau, Sagarana e Karipuna houve aumento da taxa para faixa acima de 241/100.000. Ao passo que nas TI Igarapé Lourdes Rio Negro Ocaia, Rio Branco, Roosevelt e Tubarão Latundê, houve redução na incidência (Figura 4c).

Em que pese grande flutuação observada nos indicadores ao longo do período, a análise do decênio 1997-2006 revelou que 50\% das TI em Rondônia (Sete de Setembro, Karipuna, Karitiana, Rio Negro Ocaia, Pacaas Novas, Sagarana, Igarapé Lourdes, Igarapé Lage, Uru-Eu-Wau-
Wau e Igarapé Ribeirão) apresentaram taxas brutas de TB superiores a 241/100.000 habitantes. A TI Rio Branco apresentou incidência de 121240/100.000 habitantes, enquanto as TI Tubarão Latundê, Rio Guaporé e Roosevelt apresentaram incidências na faixa de 61-120/100.000 habitantes. Nas Terras Indígenas Kaxarari, Massaco, Rio Omerê, Parque Aripuana, Kwazá do Rio São Pedro e Rio Mequens as taxas brutas foram inferiores a 31/100.000 habitantes durante todo o período analisado (Figura 4d).

\section{Incidência ajustada de tuberculose na população indígena e não indígena de Rondônia}

Com o emprego do método bayesiano local, a análise do período correspondente ao decênio 1997-2006 revelou que os municípios de Guajará-Mirim e Porto Velho permaneceram com taxas de incidência ajustadas elevadas, na faixa de 61120/100.000 habitantes. Fato semelhante ocorreu nas TI Igarapé Lage e Pacaas Novas (situadas em Guajará-Mirim) e na TI Karitiana (situada em Porto Velho), que mantiveram incidências ajustadas acima de 241/100.000.

Todavia, as incidências nas TI Rio Negro Ocaia, Sagarana, Uru-Eu-Wau-Wau e Karipuna foram atenuadas e diminuíram para faixa de 121-240/100.000 habitantes, quando comparadas com as taxas brutas que estavam acima de 
Distribuição dos casos novos de tuberculose notificados, segundo sexo, faixa etária, raça/cor e escolaridade entre indígenas e não indígenas no Estado de Rondônia, Brasil, entre 1997 e 2006.

\begin{tabular}{|c|c|c|c|c|c|}
\hline & \multicolumn{4}{|c|}{ Populações } & \multirow{3}{*}{$\begin{array}{c}\text { Total } \\
\mathbf{n}\end{array}$} \\
\hline & \multicolumn{2}{|c|}{ Indígenas } & \multicolumn{2}{|c|}{ Não indígenas } & \\
\hline & $\mathrm{n}$ & $\%$ & $\mathrm{n}$ & $\%$ & \\
\hline \multicolumn{6}{|l|}{ Sexo } \\
\hline Masculino & 157 & 56,5 & 3.131 & 62,8 & 3.288 \\
\hline Feminino & 121 & 43,5 & 1.855 & 37,2 & 1.976 \\
\hline Total & 278 & 100,0 & 4.986 & 100,0 & 5.264 \\
\hline \multicolumn{6}{|l|}{ Faixa etária (anos) } \\
\hline $0-4$ & 59 & 21,2 & 71 & 1,4 & 130 \\
\hline $5-9$ & 11 & 4,0 & 52 & 1,0 & 63 \\
\hline $10-14$ & 29 & 10,4 & 107 & 2,1 & 136 \\
\hline $15-24$ & 68 & 24,5 & 979 & 19,6 & 1.047 \\
\hline $25-34$ & 30 & 10,8 & 1.110 & 22,3 & 1.140 \\
\hline $35-44$ & 18 & 6,5 & 968 & 19,4 & 986 \\
\hline $45-54$ & 23 & 8,3 & 722 & 14,5 & 745 \\
\hline $55-64$ & 18 & 6,5 & 514 & 10,3 & 532 \\
\hline 65 e mais & 22 & 7,9 & 463 & 9,3 & 485 \\
\hline Total & 278 & 100,0 & 4.986 & 100,0 & 5.264 \\
\hline \multicolumn{6}{|l|}{ Raça/Cor } \\
\hline Branca & 1 & 0,4 & 752 & 15,1 & 753 \\
\hline Preta & 1 & 0,4 & 183 & 3,7 & 184 \\
\hline Amarela & 1 & 0,4 & 39 & 0,8 & 40 \\
\hline Parda & 7 & 2,5 & 1.325 & 26,6 & 1.332 \\
\hline Indígena & 132 & 47,5 & 7 & 0,1 & 275 \\
\hline Ignorado & 136 & 48,9 & 2.680 & 53,8 & 2.680 \\
\hline Total & 278 & 100,0 & 4.986 & 100,0 & 5.264 \\
\hline \multicolumn{6}{|c|}{ Escolaridade (anos) } \\
\hline Nenhuma & 95 & 34,2 & 897 & 18,0 & 992 \\
\hline $1-3$ & 14 & 5,0 & 548 & 11,0 & 562 \\
\hline $4-7$ & 64 & 23,0 & 2.159 & 43,3 & 2.223 \\
\hline $8-11$ & 8 & 2,9 & 699 & 14,0 & 707 \\
\hline 12 e mais & 0 & 0,0 & 169 & 3,4 & 169 \\
\hline Não se aplica & 77 & 27,7 & 225 & 4,5 & 302 \\
\hline Ignorado & 20 & 7,2 & 289 & 5,8 & 309 \\
\hline Total & 278 & 100,0 & 4.986 & 100,0 & 5.264 \\
\hline
\end{tabular}

241/100.000. Ainda assim, as taxas nas TI permaneceram pelo menos duas vezes superiores às observadas nos municípios de referência (Figura 5).

Em contrapartida, nos municípios de Cacoal, Nova Mamoré e Ji-Paraná, onde as incidências brutas foram inferiores a 60/100.000 durante todo período de estudo, após o emprego do método bayesiano as taxas ajustadas permaneceram exatamente iguais. O mesmo fenômeno foi verificado nas TI Sete de Setembro, Igarapé Ribeirão e Igarapé Lourdes onde as incidências brutas e ajustadas foram superiores a 241/100.000. Ressalta-se que o gradiente de variação entre as TI e os municípios supracitados foi ainda mais contrastante que o observado entre os municípios de Porto Velho e Guajará-Mirim (Figura 5).

\section{Discussão}

Apesar de os indígenas em Rondônia representarem menos de $1 \%$ da população do estado, no período de 1997 a 2006, eles foram responsáveis 
Figura 3

Taxa de incidência bruta de tuberculose (por 100.000 habitantes), na população não indígena, segundo municípios do Estado de Rondônia, Brasil, período de 1997 a 2006.

3a) $1997-1999$

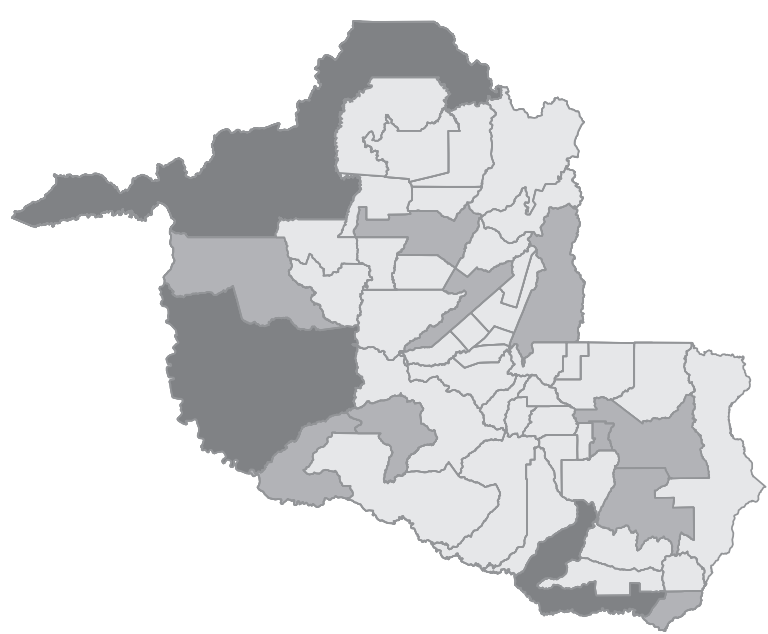

3c) $2003-2006$

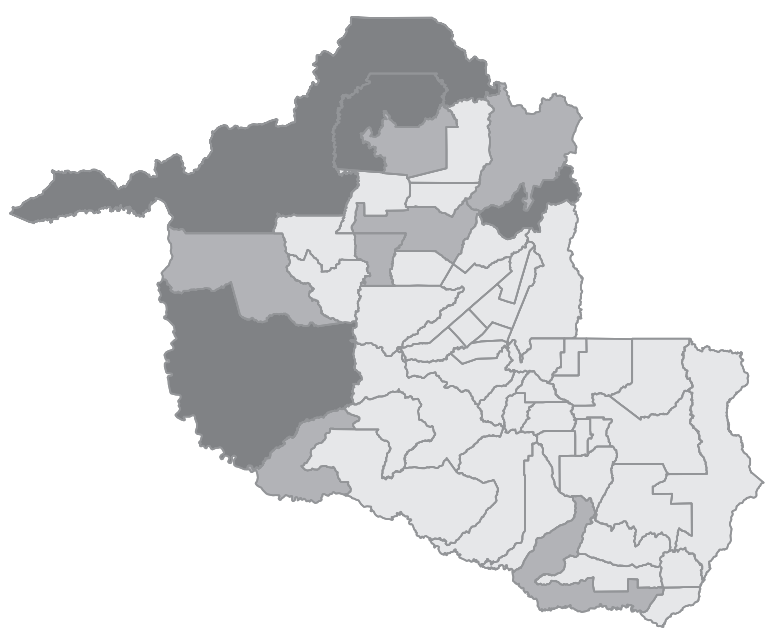

3b) $2000-2002$

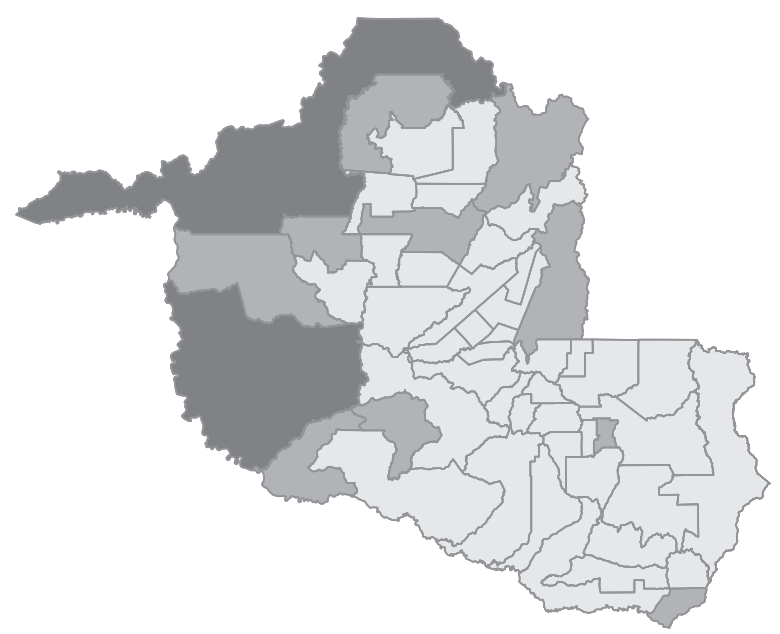

3d) $1997-2006$

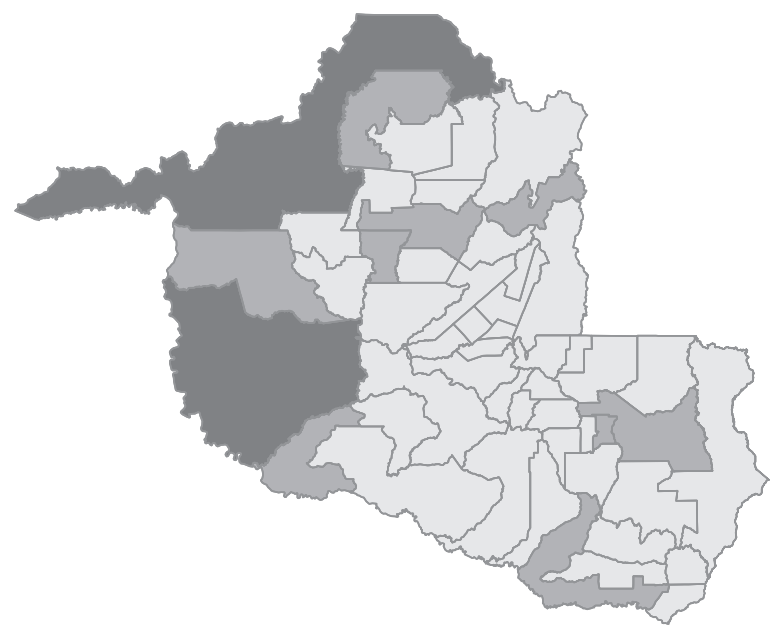

Incidência por 100.000 habitantes

$0 \sim 30$
$31 \sim 60$
$61 \sim 120$
$121 \sim 240$

$241+$

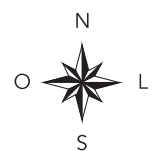

$\mathrm{s}$

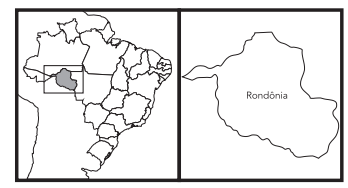

0

100

200 300 


\section{Figura 4}

Taxa de incidência bruta de tuberculose (por 100.000 habitantes) na população indígena, segundo Terras Indígenas do Estado de Rondônia, período de 1997 a 2006.

4a) $1997-1999$

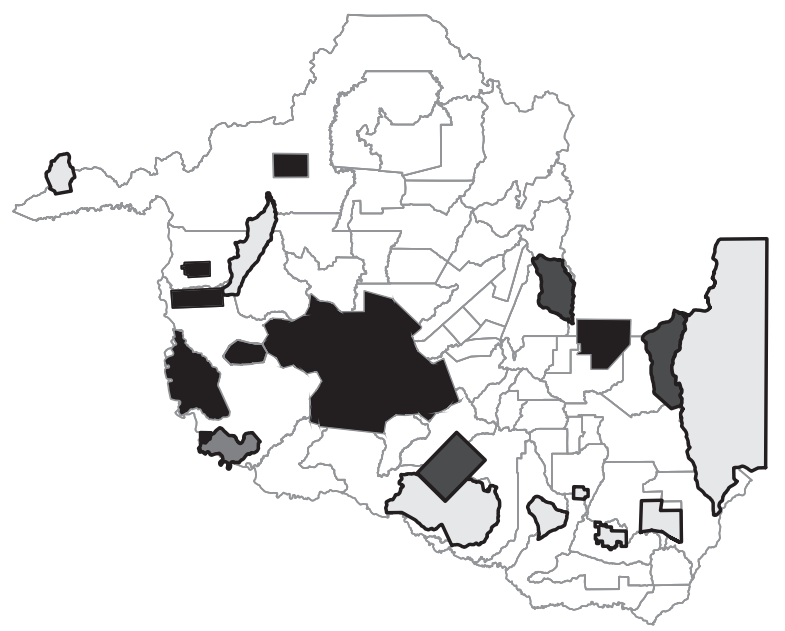

4c) $2003-2006$

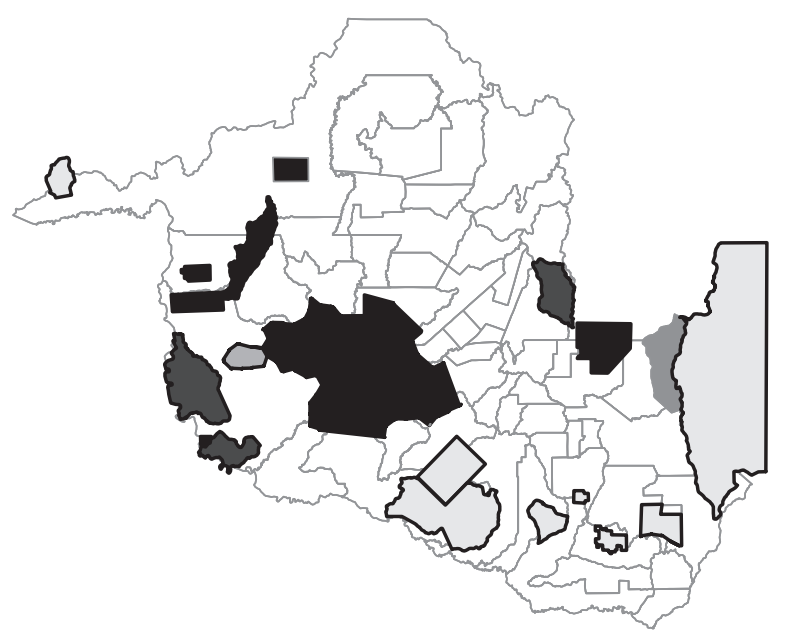

4b) $2000-2002$

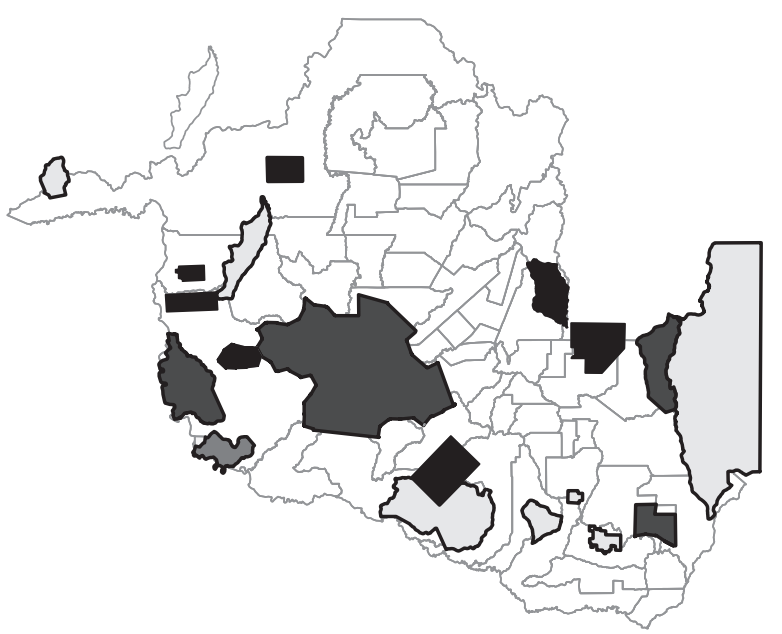

4d) $1997-2006$

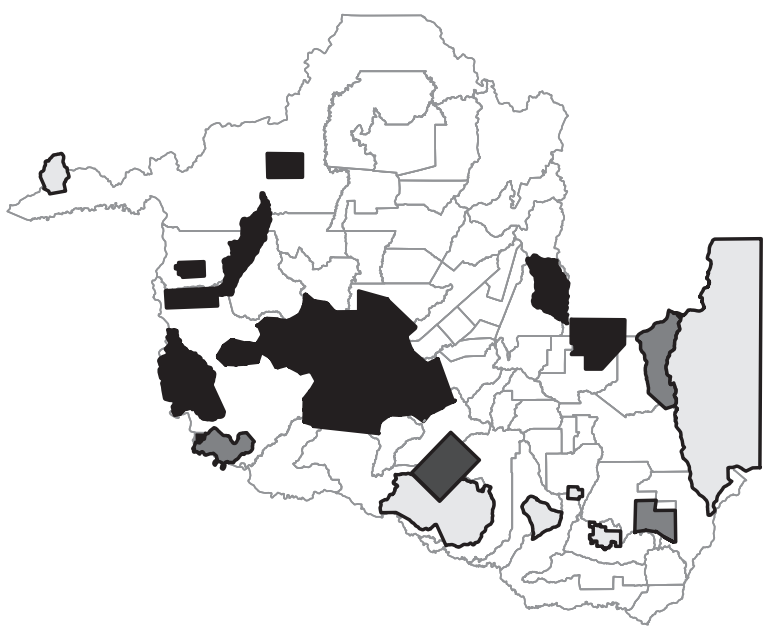

Incidência por 100.000 habitantes
$0 \sim 30$
$31 \sim 60$
$\square \quad 61 \sim 120$
$121 \sim 240$
$241+$

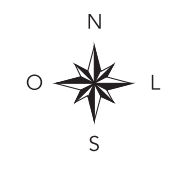

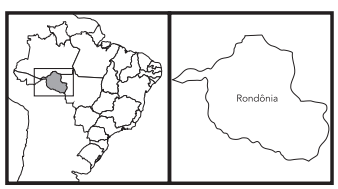

0

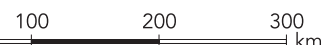


Figura 5

Taxa de incidência de tuberculose (por 100.000 habitantes), ajustada pelo método bayesiano empírico local, na população indígena e não indígena do Estado de Rondônia, Brasil, período de 1997 a 2006.

5a) $1997-1999$

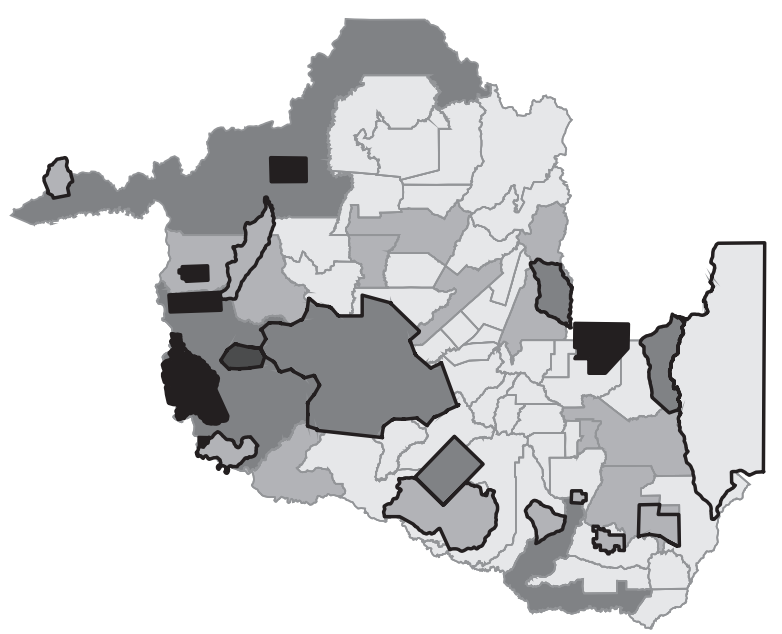

5c) $2003-2006$

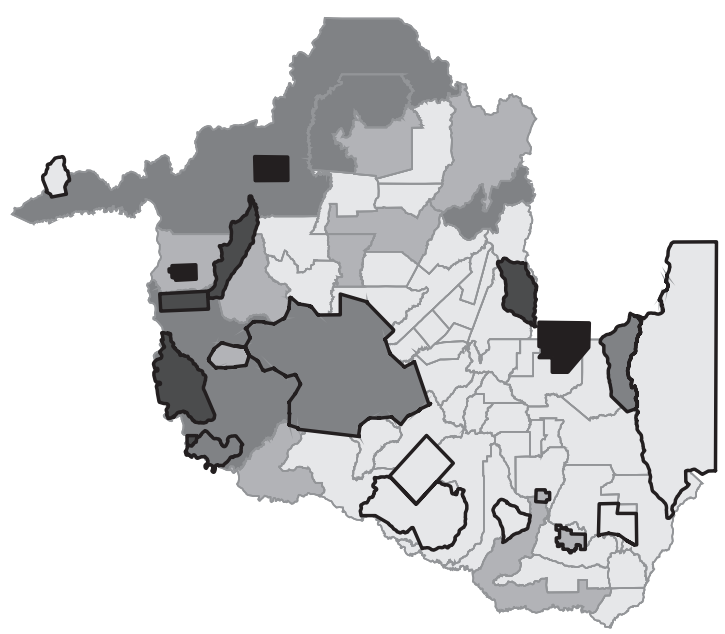

5b) $2000-2002$

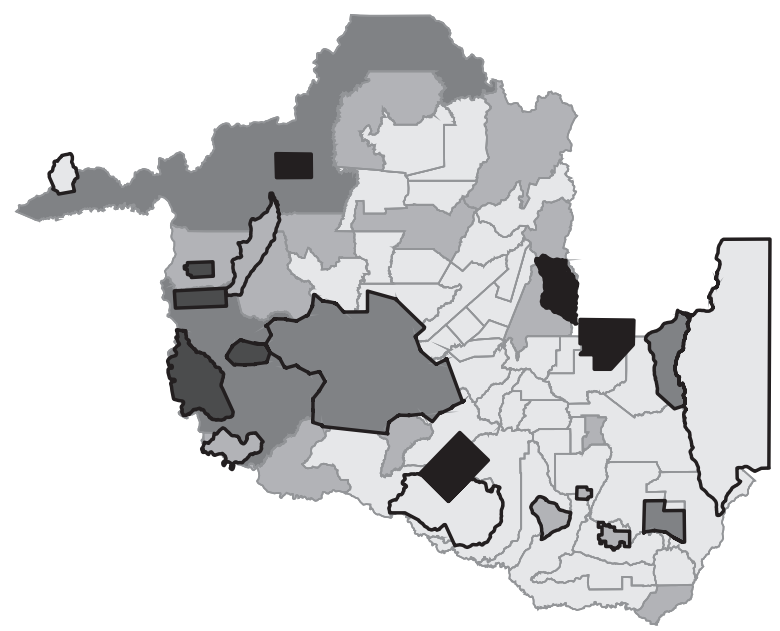

5d) $1997-2006$

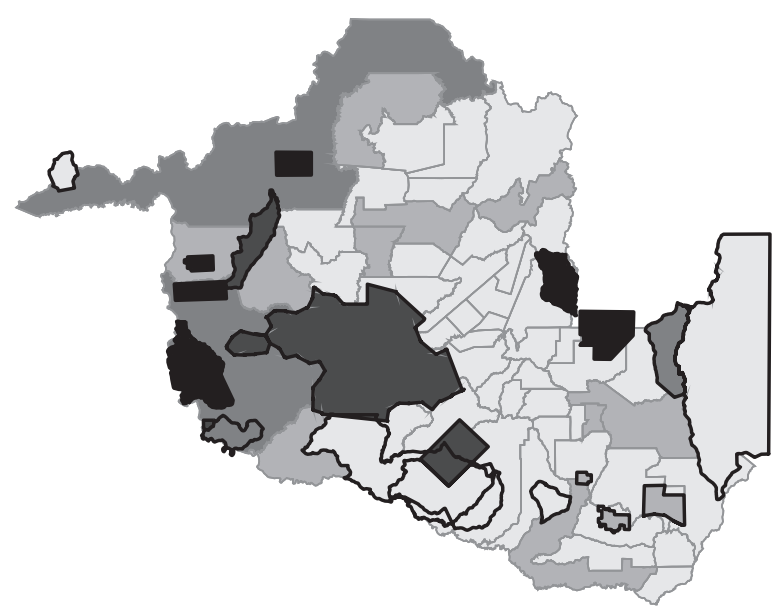

Incidência por 100.000 habitantes
$0 \sim 30$
$31 \sim 60$
$61 \sim 120$
$121 \sim 240$
$241+$
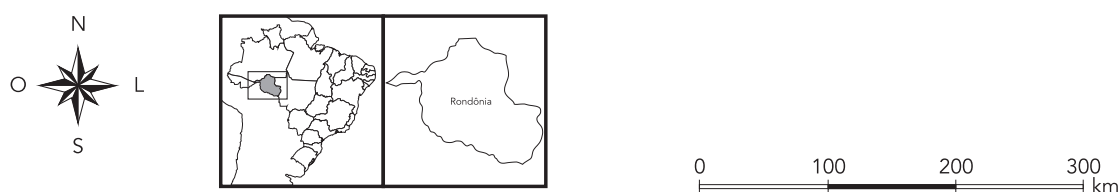
por 5\% de todos os casos novos de TB notificados. A incidência média entre os indígenas foi 415,03 , enquanto as médias em Rondônia e no Brasil ficaram em torno de 40 casos por 100.000 habitantes. Assim como descrito em estudos recentes $7,10,14,31$, nossos achados apontam a população indígena como mais vulnerável ao adoecimento por tuberculose.

No respeitante às características sociodemográficas, vale lembrar que não houve diferenças entre os achados provenientes deste estudo e os reportados na literatura nacional e internacional sobre a distribuição dos casos entre os sexos 32,33, com predominância em homens na razão de 1,3 para população indígena e 1,7 para população não indígena.

Alguns estudos demonstram associação inversa e significativa entre escolaridade e adoecimento por tuberculose 34,35 e não aderência ao tratamento ${ }^{36}$. Nesta investigação, ocorreu maior concentração de casos em pessoas com menos de sete anos de estudo, destacando-se que entre os indígenas $61,9 \%$ dos casos ocorreram em pessoas com baixo ou nenhum nível de instrução.

Frisa-se que a distribuição dos casos segundo faixa etária difere expressivamente entre indígenas e não indígenas. Para os não indígenas houve concentração de casos na faixa de 25 aos 44 anos $(41,7 \%)$, que corresponde à fase produtiva da vida, para os indígenas constatou-se maior concentração em menores de 15 anos (35,6\%). A maior proporção de casos em menores de 15 anos indica transmissão ativa e infecção recente nas aldeias, sugerindo que o controle dos contatos não está sendo realizado de forma satisfatória 9,13,37.

O preenchimento da variável raça/cor, disponível em menos de $50 \%$ dos registros, no período de estudo, mostrou-se insatisfatório e insuficiente para realizar qualquer discussão produtiva acerca de desigualdades étnicas em saúde. Para enfrentar esse problema, o procedimento de classificação dos casos indígenas baseado no nome/ sobrenome conseguiu recuperar dados perdidos e mostrou-se útil, além de permitir a construção de indicadores epidemiológicos que revelaram a magnitude da TB entre diferentes componentes étnicos da população.

No entanto, é possível que alguns indígenas que não residiam mais em aldeias ("desaldeados”), no período do estudo, tenham sido incluídos no cálculo de incidência de TB nas TI. Esse fato pode ter contribuído para superestimar a incidência de TB na população indígena. Por outro lado, também é possível que houvesse indígenas residentes em TI que não utilizavam a etnia como sobrenome, o que contribuiria para subestimar a incidência. Dessa forma, acreditamos que aconteceu um balanço entre os prováveis erros de classificação e, com isso, houve equilíbrio nas medidas, reduzindo o efeito de um provável achado espúrio.

Quando se partiu para a análise da distribuição espacial da TB no estado, as taxas brutas revelaram uma grande discrepância entre as incidências notificadas nos municípios e nas TI. Porém, após o ajuste das taxas pelo método bayesiano, detectou-se uma diminuição na heterogeneidade das medidas, sobretudo nas TI. Como exemplo, pode-se citar as TI Rio Negro Ocaia, Sagarana, Uru-Eu-Wau-Wau e Karipuna onde as incidências brutas que se situavam na faixa acima de 241/100.000 foram atenuadas e diminuíram para a faixa de 120-240/100.000 habitantes após o emprego do método bayesiano. Ainda assim, as taxas nas TI permaneceram pelo menos duas vezes superiores às observadas nos municípios de referência.

Entre alguns municípios e TI, em particular, pode-se observar discrepância ainda mais acentuada. É o caso dos municípios de Cacoal, Nova Mamoré e Ji-Paraná que apresentaram taxas ajustadas para população não indígena inferiores a 60/100.000, enquanto as taxas ajustadas das TI Sete de Setembro, Igarapé Ribeirão e Igarapé Lourdes revelaram valores acima de 241/100.000, no mesmo período.

Mediante a análise desses dados, não é possível afirmar que exista relação direta entre as taxas de incidência de TB nos municípios que abrangem TI e as taxas de incidência de TB nas TI neles contidas. Entretanto, o gradiente de incidência tão díspar reportado entre municípios e TI indica que pode haver uma exposição mais acentuada ao M. tuberculosis nas TI em comparação a outras áreas do município de referência, delineando, consequentemente, áreas de risco sanitário aumentado para a transmissão de TB.

Para além da distribuição espaço-temporal, de acordo com uma revisão sistemática da literatura realizada em 2009 38, a transmissão recente de TB está relacionada a diversos fatores, dentre os quais se destacam em ordem decrescente de associação: minorias étnicas, usuários de drogas e álcool, moradores de rua, pessoas privadas de liberdade, adultos jovens, homens portadores do HIV. Esses achados da literatura podem contribuir para explicar um pouco melhor a discrepância encontrada entre as incidências registradas em municípios e TI neste estudo.

Na perspectiva tradicional de mapeamento dos casos de TB notificados em Rondônia, terse-iam apenas quatro municípios como prioritários às ações de controle da endemia: Ariquemes, Cacoal, Guajará-Mirim e Porto Velho. Vale lembrar que em Ariquemes não existem TI. 
Todavia, quando são incorporadas as TI, pôdese observar claramente que as áreas de risco se ampliaram e modificaram sua configuração. Acrescido a isso, o fato de os limites das TI não coincidirem com os limites territoriais dos municípios e do estado impõe importantes desafios para o desenvolvimento das ações de controle da TB em Rondônia.

Como indicam Souza et al. 18,19, quando se busca conhecer a distribuição de determinados agravos num espaço geográfico específico, é necessário considerar unidades básicas menores para coleta e análise de dados com a intenção de vislumbrar áreas ou situações de risco. Esse processo foi visivelmente exemplificado neste trabalho quando se mudou a unidade de análise para as TI.

De toda forma, o Sistema de Informação Geográfica (SIG) não substitui outros métodos convencionais utilizados pelo PNCT para a análise da situação da TB numa dada localidade. Contudo, tal investigação mostrou a importância do geoprocessamento na identificação de áreas de maior risco de transmissão de tuberculose.

Segundo Garnelo et al. 23, o SIG é uma ferramenta útil para a gestão, possibilitando análises de situações sanitárias, avaliação de risco populacional, construção de cenários que viabilizem o planejamento de estratégias de intervenção em diversos níveis, transitando com rapidez e eficiência entre macro e micro realidades, e possibilitando a expressão da diversidade etnoepidemiológica que se oculta nas abordagens totalizantes dos sistemas convencionais de informação em saúde.

Cumpre destacar que alguns municípios e TI não notificaram casos de TB em todo o período analisado. Nesse sentido, Braga 21 ressalta que algumas hipóteses têm sido geradas pela avaliação do comportamento epidemiológico da TB. Se a TB é determinada por precárias condições socioeconômicas, espera-se que o baixo nível de vigilância dos serviços oferecidos a determinados grupos populacionais seja uma explicação plausível para a inexistência de casos notificados. Nesse contexto, é necessária uma atenção especial a esses municípios e TI “silenciosos”, pois eles podem apresentar alta carga da doença e não estar sendo reconhecidos como prioritários.

Considerando o período como um todo, após o uso do método bayesiano empírico local, foi possível verificar que, das oito TI sem ocorrência de casos, quatro foram incluídas na faixa de 1 a 30 e três na faixa de 31 a 60 casos/100.000 habitantes. Por outro lado, a TI Karipuna que possuía taxa bruta média acima de 3.000 (mesmo que nos dois períodos iniciais não tenham sido notificados casos), foi classificada na faixa de 121 a 240 casos, tendo sua incidência corrigida para $137,28 / 100.000$ habitantes. Portanto, a estimativa bayesiana é uma ferramenta de grande valia quando se analisam dados provenientes de áreas com elevado número de casos e áreas "silenciosas”. Deve-se considerar que esse procedimento também pode gerar uma informação equivocada, caso efetivamente não haja transmissão nas áreas sem registro de casos.

Salienta-se-se que estudos que utilizam dados secundários de notificação, em geral, apresentam como limitações problemas relacionados à cobertura, à oportunidade e ao sub-registro de casos. Além disso, a coleta não sistemática de informações e a baixa representatividade dos povos indígenas nos censos demográficos nacionais e nos sistemas nacionais de informação em saúde podem contribuir para enviesar as estimativas de incidência apresentadas neste trabalho.

Vale lembrar que para o cálculo das taxas ajustadas, nos municípios próximos aos contornos de Rondônia, não foram considerados os casos de TB nem a população fora dos limites do estado, tanto em áreas de fronteira nacional quanto internacional, o que também pode ter contribuído para enviesar as estimativas.

Apesar dessas limitações, os dados de morbidade por TB confirmaram que a cobertura e a qualidade do SINAN em Rondônia permitiram a realização de análises epidemiológicas detalhadas, comparando regiões geográficas e diferentes grupos populacionais. Essas informações podem embasar a tomada de decisões gerenciais por parte do PNCT, em seus níveis nacional, estadual e municipal.

O emprego do método bayesiano empírico local, com base nos valores da vizinhança, diminuiu a heterogeneidade espacial das taxas, mas importa salientar a necessidade da incorporação de métodos de modelagem estatística espacial para verificação de possíveis variáveis definidoras de situações coletivas de risco para TB em diferentes áreas de estudo.

Sugere-se que os métodos empregados neste estudo sejam replicados em outros locais, a fim de contribuir para melhor compreensão dos mecanismos envolvidos na distribuição espacial da TB e adaptar as ações desenvolvidas pelo PNCT à realidade das sociedades indígenas brasileiras.

Por fim, os achados provenientes desta investigação permitiram refinar o foco de atenção para áreas prioritárias que carecem de intervenção, demonstrando claramente que os povos indígenas no Estado de Rondônia requerem maior atenção no que se refere ao adequado controle da TB. 


\section{Resumo}

Este estudo analisou a distribuição espacial e temporal das incidências brutas e ajustadas de tuberculose (TB) no período 1997-2006, identificando áreas de maior risco para indígenas e não indígenas de Rondônia, Brasil. Foi realizado estudo ecológico, tendo como unidades de análise municípios e Terras Indígenas (TI), utilizando método bayesiano empírico local para ajuste das taxas. A incidência média bruta de TB para não indígenas foi 35,6/100.000 habitantes, enquanto para indígenas foi 415,0/100.000 habitantes. As TI Karipuna, Sete de Setembro, Igarapé Ribeirão e Karitiana apresentaram incidência $>600 / 100.000$. Observou-se nos indígenas maior número de casos em $<15$ anos e com baixa escolaridade, diferente do ocorrido nos não indígenas. Após ajuste, as taxas em algumas TI ultrapassaram 240/100.000, enquanto, nos municípios coincidentes com as TI, elas ficaram entre 61 120/100.000 habitantes. O método bayesiano diminuiu a heterogeneidade das taxas. Evidências apontam os indígenas como grupo mais vulnerável ao adoecimento e mostram áreas que requerem maior atenção para adequado controle da TB em Rondônia.

Tuberculose; Análise Espacial; Vigilância em Saúde; Índios Sul-Americanos

\section{Referências}

1. Instituto Socioambiental. Povos indígenas no Brasil: 2001-2005. São Paulo: Instituto Socioambiental; 2006.

2. Coordenação Geral de Planejamento e Avaliação, Fundação Nacional de Saúde. Relatório de gestão 2008. Brasília: Ministério da Saúde/Fundação Nacional de Saúde; 2009.

3. Ministério da Saúde. Portaria no 2.656, de 17 de outubro de 2007. Dispõe sobre as responsabilidades na prestação da atenção à saúde dos povos indígenas, no Ministério da Saúde e regulamentação dos Incentivos de Atenção Básica e Especializada aos Povos Indígenas. Diário Oficial da União 2007; 18 out.

4. Santos RV, Coimbra Jr. CEA. Cenários e tendências da saúde e da epidemiologia dos povos indígenas no Brasil. In: Coimbra Jr. CEA, Santos RV, Escobar AL, organizadores. Epidemiologia e saúde dos povos indígenas no Brasil. Rio de Janeiro: Editora Fiocruz; 2003. p. 13-47.

\section{Colaboradores}

P. C. Basta participou da concepção e projeto, análise e interpretação dos dados, redação do artigo e aprovação final da versão a ser publicada. T. E. M. P. Melo, A. P. C. Resendes e R. Souza-Santos contribuíram com a análise e a interpretação dos dados, redação do artigo e aprovação final da versão a ser publicada.

\section{Agradecimentos}

À Wandcliuce Melo Pinheiro, chefe do núcleo de Hanseníase e Tuberculose (NDPSHT/GTVAE/AGEVISA/RO), por possibilitar o acesso ao banco de dados utilizado nesta pesquisa. A Coordenação Regional da FUNASA em Rondônia pelo acesso à base de dados do Sistema de Informação da Atenção à Saúde Indígena. Ao colega Linconl Uchôa Sidon pelo auxílio na estruturação do banco de dados. Ao CNPq, processo 402505/2008-5, pelo apoio financeiro. Ao Departamento de Ciência e Tecnologia do Ministério da Saúde (DECIT/MS) pelo prêmio de incentivo em ciência e tecnologia para o SUS 2010, categoria especialização.
5. Secretaria Executiva, Ministério da Saúde. Programa Saúde Indígena: etnodesenvolvimento das sociedades indígenas. Brasília: Ministério da Saúde; 2001.

6. Amarante JM, Costa VLA. A tuberculose nas comunidades indígenas brasileiras na virada do século. Bol Pneumol Sanit 2000; 8:5-12.

7. Escobar AL, Coimbra Jr. CEA, Camacho LAB, Portela MC. Tuberculose em populações indígenas de Rondônia, Amazônia, Brasil. Cad Saúde Pública 2001; 17:285-98.

8. Marques AMC, Cunha RV. A medicação assistida e os índices de cura de tuberculose e de abandono de tratamento na população indígena GuaraníKaiwá no município de Dourados, Mato Grosso do Sul, Brasil. Cad Saúde Pública 2003; 19:1405-11.

9. Basta PC, Coimbra Jr. CEA, Escobar AL, Santos RV. Aspectos epidemiológicos da tuberculose na população indígena Suruí, Amazônia, Brasil. Rev Soc Bras Med Trop 2004; 37:338-42. 
10. Levino A, Oliveira RM. Tuberculose na população indígena de São Gabriel da Cachoeira, Amazonas, Brasil. Cad Saúde Pública 2007; 23:1728-32.

11. Machado Filho AC. Incidência da tuberculose em indígenas do município de São Gabriel da Cachoeira, AM. Rev Soc Bras Med Trop 2008; 41:243-6.

12. Bóia MN, Carvalho FA, Sodré FC, Porras-Pedroza BE, Faria EC, Magalhães GAP, et al. Tuberculose e parasitismo intestinal em população indígena na Amazônia brasileira. Rev Saúde Pública 2009; 43:176-8.

13. Basta PC, Rios DPG, Alves LCC, Sant'Anna CC, Coimbra Jr. CEA. Estudo clínico-radiológico de crianças e adolescentes indígenas Suruí, Região Amazônica. Rev Soc Bras Med Trop 2010; 43:719-22.

14. Basta PC, Coimbra Jr. CEA, Welch JR, Alves LCC, Santos RV, Camacho LAB. Tuberculosis among the Xavante Indians of the Brazilian Amazon: an epidemiological and ethnographic assessment. Ann Hum Biol 2010; 37:643-57.

15. Programa Nacional de Controle da Tuberculose, Secretaria de Vigilância em Saúde, Ministério da Saúde. Manual de recomendações para o controle da tuberculose no Brasil. Brasília: Ministério da Saúde; 2010.

16. Basta PC, Coimbra Jr. CEA, Escobar AL, Santos RV, Alves LCC, Fonseca LS. Survey for tuberculosis in an indigenous population of Amazonia: the Suruí of Rondônia, Brazil. Trans R Soc Trop Med Hyg 2006; 100:579-85.

17. Basta PC, Coimbra Jr. CEA, Alves LCC. Padrões radiológicos da tuberculose pulmonar em indígenas Suruí de Rondônia, Amazônia. Rev Soc Bras Med Trop 2006; 39:221-3.

18. Souza WV, Ximenes RAA, Albuquerque MFM, Lapa TM, Portugal JL, Lima MLC, et al. The use of socioeconomic factors in mapping tuberculosis risk areas in a city of northeastern Brazil. Rev Panam Salud Pública 2000; 8:403-10.

19. Souza WV, Albuquerque MFM, Barcellos CC, Ximenes RAA, Carvalho MS. Tuberculose no Brasil: construção de um sistema de vigilância de base territorial. Rev Saúde Pública 2005; 39:82-9.

20. Souza WV, Carvalho MS, Albuquerque MFM, Barcellos CC, Ximenes RAA. Tuberculosis in intra-urban settings: a Bayesian approach. Trop Med Int Health 2007; 12:323-30.

21. Braga JU. Vigilância epidemiológica e o sistema de informação da tuberculose no Brasil, 2001-2003. Rev Saúde Pública 2007; 41 Suppl 1:77-88.

22. Sales CMM, Figueiredo TAM, Zandonade E, Maciel ELN. Análise espacial da tuberculose infantil no estado do Espírito Santo, 2000 a 2007. Rev Soc Bras Med Trop 2010; 43:435-9.

23. Garnelo L, Brandão LC, Levino A. Dimensões e potencialidades dos sistemas de informação geográfica na saúde indígena. Rev Saúde Pública 2005; 39:634-40.

24. Coordenação Geral de Doenças Endêmicas, Área Técnica de Pneumologia Sanitária, Departamento de Vigilância Epidemiológica, Secretaria de Vigilância em Saúde, Ministério da Saúde. Guia para cálculo de indicadores básicos e de avaliação da base de dados de tuberculose do Sistema de Informação de Agravos de Notificação - SINAN. Brasília: Ministério da Saúde; 2004.
25. Bhopal R. Ethnicity, race, and health in multicultural societies: foundations for better epidemiology, public health, and health care. Oxford: Oxford University Press; 2007.

26. Imbiriba EB, Basta PC, Pereira ES, Levino A, Garnelo L. Hanseníase em populações indígenas do Amazonas, Brasil: um estudo epidemiológico nos municípios de Autazes, Eirunepé e São Gabriel da Cachoeira (2000 a 2005). Cad Saúde Pública 2009; 25:972-84.

27. Landis JR, Koch GG. The measurement of observer agreement for categorical data. Biometrics 1977; 33:159-74.

28. Instituto Brasileiro de Geografia e Estatística. Tendências demográficas: uma análise dos indígenas com base nos resultados da amostra dos censos demográficos de 1991 e 2000. Rio de Janeiro: Instituto Brasileiro de Geografia e Estatística; 2005.

29. Mason TJ, Mckay FW, Hoover R, Blot WJ, Fraumenti Jr. JF. Atlas of cancer mortality among U.S. Nonwhites: 1950-1969. Washington DC: Department of Health, Education, and Welfare, U.S. Government Printing Office; 1976.

30. Mason TJ, Mckay FW, Hoover R, Blot WJ, Fraumenti Jr. JF. Atlas of cancer mortality for U.S. Counties, 1950-1969. Washington DC: Department of Health, Education, and Welfare, U.S. Government Printing Office; 1975.

31. Coimbra Jr. CEA, Basta PC. The burden of tuberculosis in indigenous peoples in Amazonia, Brazil. Trans R Soc Trop Med Hyg 2007; 101:635-6.

32. World Health Organization. Global tuberculosis control: epidemiology, strategy, financing: WHO report 2009. Geneva: World Health Organization 2009. http://www.who.int/tb/publications/glob al_report/2009/pdf/full_report.pdf (acessado em $25 / \mathrm{Fe} / 2011$ ).

33. Sociedade Brasileira de Pneumologia e Tisiologia. III diretrizes para tuberculose da Sociedade Brasileira de Pneumologia e Tisiologia. J Bras Pneumol 2009; 35:1018-48.

34. Menezes AMB, Costa JD, Gonçalves H, Morris S, Menezes M, Lemos S, et al. Incidência e fatores de risco para tuberculose em Pelotas, uma cidade do Sul do Brasil. Rev Bras Epidemiol 1998; 1:50-60.

35. Severo NPF, Leite CQF, Capela MV, Simões MJS. Características clínico-demográficas de pacientes hospitalizados com tuberculose no Brasil, no período de 1994 a 2004. J Bras Pneumol 2007; 33:565-71.

36. Ferreira SMB, Silva AMC, Botelho C. Abandono do tratamento da tuberculose pulmonar em Cuiabá MT - Brasil. J Bras Pneumol 2005; 31:427-35.

37. Natal S. Tuberculose na criança. Bol Pneumol Sanit 2000; 8:20-5.

38. Nava-Aguilera E, Andersson N, Harris E, Mitchel S, Hamel C, Shea B, et al. Risk factors associated with recent transmission of tuberculosis: systematic review and meta-analysis. Int J Tuberc Lung Dis 2009; 13:17-26.

Recebido em 31/Mai/2011

Versão final reapresentada em 22/Ago/2011 Aprovado em 13/Set/2011 